\title{
The Enhancement of BAO in the SDSS MGS
}

\author{
H. J. Tian ${ }^{1}$, M. C. Neyrinck ${ }^{2}$, T. Budavári ${ }^{2}$ and A. S. Szalay ${ }^{2}$ \\ ${ }^{1}$ China Three Gorges University, Yichang, 443002, China; hjtian@lamost.org \\ ${ }^{2}$ Department of Physics and Astronomy, Johns Hopkins University, Baltimore, MD 21218
}

\begin{abstract}
We show that redshift-space distortions of galaxy correlations have a strong effect on correlation functions with the signature of the Baryon Acoustic Oscillations (BAO). Near the line of sight, the features become sharper as a result of redshift-space distortions. We analyze the SDSS DR7 main-galaxy sample (MGS), splitting the sample into slices $2.5 \mathrm{deg}$ on the sky in various rotations. Measuring $2 \mathrm{D}$ correlation functions in each slice, we do see a sharp bump along the line of sight. Using Mexican-hat wavelets, we localize it to $(110 \pm 10) h^{-1} \mathrm{Mpc}$ and estimate its significance at about $4 \sigma$.
\end{abstract}

Keywords. cosmology: large-scale structure of Universe - methods: data analysis

\section{BAO in the SDSS MGS}

BAO has been typically characterized by the observations(Eisenstein et al. 2005, Cole et al. 2005). Correlation functions of lower-dimensional subsets for a homogeneous isotropic random field are identical to the one estimated from the full 3-dimensional one.

We build a sample of SDSS DR7 MGS (Strauss et al. 2002) galaxies, and analyze the LOS and 2D correlation functions (Fig. 1) using thus a methodology: we subdivide the sample into many $2.5 \mathrm{deg}$ on the sky in various rotations slices, compute the $2 \mathrm{D}$ redshift space correlation function and calculate the average (Tian et al. 2011).
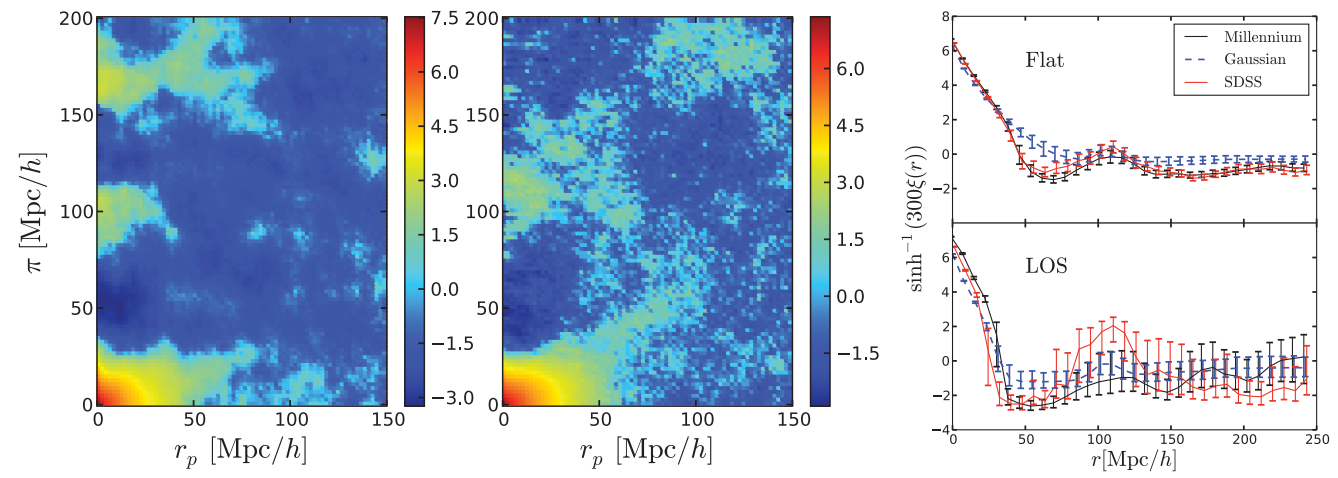

Figure 1. The 2-D and 1-D correlation functions. The left panel is measured from the $100-750 h^{-1} \mathrm{Mpc}$ full SDSS MGS sample, and the middle is from the $300-750 h^{-1} \mathrm{Mpc}$ high- $z$ sample (excluding the Sloan Great Wall, which alters clustering statistics substantially). The right-top panel shows the $\xi$ averaged uniformly over angle from the simulations and the high- $z$ SDSS sample, and the right-bottom uses only data within 6 deg of the LOS.

\section{References}

Eisenstein, D. J. et al. 2005, ApJ, 633, 560

Cole, S. et al. 2005, MNRAS, 362, 505

Strauss, M. A. et al. 2002, AJ, 124, 1810

Tian, H. J. et al. 2011, ApJ, 728, 34 